\title{
Physiological mechanisms behind the effectiveness of Pranayama
}

\author{
A G Ramakrishnan \\ MILE Lab, Dept of Electrical Engineering, Indian Institute of Science, Bangalore
}

\begin{abstract}
:
The benefits of pranayama for positive health are well known. Even though there are many studies published on the effectiveness of pranayama, there are very few papers, which actually have systematically studied the physiological mechanisms involved, causing the benefits of pranayama, especially with respect to the cardiac function. This article attempts to have a detailed look at the physiology behind deep breathing. The article also conjectures that voluntary, deep breathing with attention may have a role to play in faster recovery from surgeries, and prevent or delay the onset of Alzheimer's disease, Parkinson's disease and may be, even cancer. Detailed, controlled studies are needed to prove or disprove these conjectures.
\end{abstract}

Key words: pranayama, oxygen, carbon-dioxide, $\mathrm{pH}$, chemoreceptors, Parkinson's disease, Alzheimer's disease, cancer, wound healing, respiratory sinus arrhythmia, CSF, vital capacity.

\section{Introduction}

Indian tradition has always extolled the practice of different types of deep breathing techniques, generally known as pranayama for better health of a human being. A number of studies have shown reduction of heart rate and blood pressure after different types of pranayama practices [1, 2]. However, not many have looked into the mechanism of how deep breathing impacts the various physiological processes, leading to positive health, especially that of the heart [3].

In this article, we have carefully studied the respiratory physiology and the cardio-respiratory control circuits and we propose possible mechanisms behind the effect of deep breathing in enhancing cardiac health. We also establish that voluntary, attentive, deep breathing is a very effective cardiac exercise.

\section{The muscles involved in inspiration:}

Breathing has not been specifically looked at as a cardiac exercise. However, this is because of a lack of focused study or observation of the physiology involved. Normal, involuntary inspiration is primarily caused by the contraction of the eleven pairs of external intercostal muscles and the diaphragm, which add up to 23 muscles. The sternocleidomastoid (left and right) and the scalene muscles (anterior, middle and posterior scalene) are considered accessory muscles of breathing. They assist in elevating the rib cage. These five muscles get involved depending upon the level of effort put in by the subject during inspiration. Thus, deep inspiration in yogic breathing can involve up to 28 muscles. 


\section{The muscles involved in expiration:}

Normal expiration is passive, accomplished by the recoil of the thoracic cage and may hardly involve any muscle contraction. However, conscious, deep expiration is accomplished by the contraction of the eleven pairs of internal intercostal muscles, the eleven pairs of innermost intercostal muscles and the abdominal (rectus abdominus, internal and external obliques and transverse abdominus) muscles. The internal intercostal muscles can add force to the exhalation process by lowering the rib cage. The four abdominal muscles can press the abdominal organs upward into the diaphragm, thus diminishing the volume of the thoracic cavity. These abdominal muscles are the main ones involved, when one performs the quick exhalations in kapala bhati. Kapala bhati is a specific type of breathing suggested in Gheranda Samhita [4], where the exhalation is active and the inhalation is passive, the opposite of normal breathing. Thus, deep, active expiration in pranayama can recruit up to 48 muscles.

In addition to the above muscles, the following 13 muscles have also been observed to contribute to respiration:serratus anterior, pectoralis major and pectoralis minor, trapezius, latissimus dorsi, erector spinae iliocostalis lumborum, quadratus lumborum, serratus posterior superior, serratus posterior inferior, levatores costarum, transversus thoracis, subclavius [5]. Thus, by forced inhalation and exhalation, one can activate 89 muscles in all - which is nearly $14 \%$ of all the muscles in the body!

Deep breathing is normally activated by the autonomic nervous system during strenuous exercises such as aerobic exercise, climbing a steep mountain or sprinting. This is because the increased muscle activity demands increased oxygen. However, interestingly, it is the brain, rather than the skeletal muscles, which consume more oxygen in general. Even though the adult brain weighs less than $2 \%$ of the body weight, it consumes $20 \%$ of the oxygen inhaled by the human beings. Since most of our occupations today involve brain work, rather than physical work, it is important to delve deeper into the physiological and control mechanisms of breathing and the role of the latter in ensuring efficient functioning of the brain.

\section{Neural control of the respiratory muscles:}

These muscles are controlled by 18 ( 5 cervical, 12 thoracic and one lumbar) pairs of the total 31 pairs of the spinal nerves! The accessory muscles are controlled by the three pairs of cervical nerves $\mathrm{C} 1$ to $\mathrm{C} 3$, and the diaphragm is controlled by $\mathrm{C} 3$ to $\mathrm{C} 5$. The eleven pairs of thoracic nerves T1 to T11 are responsible for the contraction of the 11 pairs each of the external, internal and the innermost intercostal muscles. The abdominal muscles are innervated by the thoracic nerves T6 to T12 and the lumbar nerve L1. The involvement of 36 spinal nerves in breathing clearly shows the importance given to respiration by nature.

\section{Respiratory Sinus Arrhythmia:}

Respiratory sinus arrhythmia (RSA) is a physiological phenomenon reflecting respiratorycirculatory interactions universally observed among vertebrates. Every time one inhales, the heart rate (HR) goes up. Every time one exhales, the HR goes down. It is thought that nature evolved this mechanism to increase the effectiveness of perfusion during inhalation and save energy expenditure during exhalation. Thus, because of the RSA alone, active, deep respiration constantly exercises the cardiac muscle, alternately increasing and decreasing the 
HR. Let us not forget that our circulatory system takes the increased Oxygen to each of our 130 Trillion cells in every cardiac cycle! Thus, conscious deep breathing is an amazing cardiorespiratory exercise!

\section{$900 \%$ increase is possible from the tidal volume to the vital capacity!}

In normal (involuntary, passive) breathing, the volume of air displaced between inhalation and exhalation (called the tidal volume) is about $500 \mathrm{ml}$ or $7 \mathrm{~mL} / \mathrm{kg}$ of body mass for a healthy, young adult. With deep breathing with maximal effort, one can reach the vital capacity (maximum amount of air that can be moved in and out of the lungs in a respiratory cycle) of about 4.8 litres per respiration.

This is almost 10 times the tidal volume or a $900 \%$ increase in the effectiveness of breathing OR the volume of air displaced per breathing cycle. Together with holding the breath after inhalation taught as part of advanced pranayama practices, this can ensure extremely effective gas exchange, better eliminating the carbon-dioxide from the body!

\section{Involvement of chemoreceptors, baroreceptors and glands:}

Daly and Bondurant [6] showed that breathing pure oxygen decreases the heart rate. Deep breathing naturally results in increased oxygen and reduced carbon-dioxide levels in the blood and consequently, reduced heart rate and pumping force of the heart due to the feedback given by the chemoreceptors at the aorta and carotid artery to the cardiorespiratory control centres located in the pons and medulla oblongata in the brainstem. The baroreceptors in the carotid sinus and the aortic arch constantly sense the changes in the blood pressure by sensing the changes to the tension of the arterial walls. The adrenal and the thyroid glands can also change the heart rate and blood pressure, by controlling the release of epinephrine and thyroid harmones. Thus, the whole process involves the complex feedback circuitry involving the baroreceptors, chemoreceptors and hormones. Thus, deep breathing is a very effective cardiac exercise!

\section{Role of oxygen in wound healing:}

Wounds need oxygen to heal. Continuous supply of oxygen to the tissue through microcirculation is vital for the healing process and for resistance to infection [7]. One of the biggest factors that can inhibit the body's ability to recover is low oxygen flow to the affected area. Hence, the evaluation of tissue perfusion and oxygenation is important in all types of wounds. The Johns Hopkins hospital uses hyperbaric oxygen (HBO) therapy for wound healing. We normally think of cuts, falls and accidents when we hear the word 'wound'. However, all surgeries result in wounds that take time to heal. Thus, it is the conjecture of the author that recovery from surgeries may be accelerated by deep breathing.

\section{Oxygen and cancer connection:}

The Nobel-prize winning biochemist Dr. Otto Heinrich Warburg $[8,9]$ showed that cancer basically needs a low oxygen environment to survive. He suggested that cancer cells "live in hypoxic, very low oxygen, and acidic conditions and derive energy from sugars by fermenting 
them the way yeast does." From this, he theorized that these low-oxygen and highly-acidic conditions caused cancer. Thus, it is the author's conjecture that when the body cells have sufficient oxygen, cancer may be prevented or at least delayed! Hyperbaric oxygen has been shown to induce an antiangiogenic effect in two mammary tumor models, in addition to one glioma model.

\section{Modulation of CSF circulation by respiration:}

Beta amyloid is produced as a waste product of metabolic activity in the brain. It is a toxic protein associated with Alzheimer's disease [10]. In Alzheimer's disease, beta-amyloid clumps together to form amyloid plaques, negatively impacting communication between neurons. Boosting CSF has also been linked to improvement in cognitive function and reduction of cognitive concerns in the elderly [11]. It is that author's conjecture that improved circulation of CSF in the brain may aid in the effective elimination of metabolic waste products such as beta-amyloids from the brain, thus reducing the risk of Alzheimer's disease. A very recent study [12] has shown that breathing modulates the circulation of cerebrospinal fluid (CSF) in the brain. This is a finding with far-reaching implications. The author conjectures that this implies that with regular practice of deep breathing, one may be able to improve the circulation of CSF in the brain and either prevent or delay the onset of Alzheimer's disease.

\section{Can deep breathing delay the onset of Parkinson's?}

Parkinson's disease involves loss of dopaminergic neurons in Substantia Nigra pars compacta (SNc) [13]. Due to their structural and functional properties, neurons in the SNc are one of the most vulnerable and energy consuming neurons. It has been proposed that the primary factor that causes the degeneration of SNc neurons is their high metabolic requirements [14]. A very recent paper [15], based on computational modelling of basal ganglia, suggests that this process might be initiated by weak-excitotoxicity mediated by energy deficit.

The author conjectures that regular, voluntary deep breathing can increase the supply of oxygen to the brain, thus preventing or delaying the onset of Parkinson's disease. Once the supply of nutrients is sufficient, the body has adequate mechanisms in terms of feedback and preferential supply, to ensure that the needs of structures that demand additional nutrition are met.

\section{Gene expression changes due to pranayama:}

Epigenetics offers new possibilities to the humanity, which earlier believed that one's destiny is fully controlled by one's genes, which completely decided whether one will develop a disease or not. Using a controlled study, Su Qu [16] showed that two hours of Sudarshan Kriya and related practices can cause changes in the expression of 111 genes in the peripheral blood mononuclear cells (PBMCs) in healthy people, as compared to only 38 genes in the case of people, who had a nature walk and listened to relaxing music. More such studies are needed to explore the exciting possibilities that epigenetics promise. It goes without saying that it is ultimately important to look at the physiological and the molecular mechanisms that lead to such alterations in gene expression profiles in the PBMCs. 


\section{Conclusion:}

Active, deep pranayamic breathing is a very effective, cardiorespiratory exercise, with a potential to recruit up to 89 muscles, 36 spinal nerves and a number of peripheral and central chemoreceptors, volume receptors, besides possibly the adrenal and thyroid glands (in releasing epinephrine and thyroid hormones). Thus, the health of the heart improves, and the heart rate and the blood pressure are bound to reduce with prolonged practice of pranayama. If the recent findings of CSF flow modulation by respiration are confirmed, then regular deep breathing has the potential to provide sufficient supplies and also delay or prevent the accumulation of beta amyloids in the brain, thus preventing Parkinson's and Alzheimer's diseases.

\section{References:}

1. Pramanik, Tapas, Hari Om Sharma, Suchita Mishra, Anurag Mishra, Rajesh Prajapati, and Smriti Singh. "Immediate effect of slow pace bhastrika pranayama on blood pressure and heart rate." The Journal of Alternative and Complementary Medicine 15, no. 3 (2009): 293295.

2. Dhungel, K. Upadhyay, V. Malhotra, D. Sarkar, and R. Prajapati. "Effect of alternate nostril breathing exercise on cardiorespiratory functions." Nepal Med Coll J 10, no. 1 (2008): 25-27.

3. Sengupta, Pallav. "Health impacts of yoga and pranayama: A state-of-the-art review." International journal of preventive medicine 3, no. 7 (2012): 444.

4. Gheranda, "The Gheranda Sanhita: A Treatise on Hatha Yoga," Translated by Rai Bahadur Srisa Chandra Vasu, Bombay Theosophical fund, 1895.

5. Kendall, F., McCreary, E., Provance, P., Rodgers, M., Romai, W. (2005). Muscles testing and function with posture and pain (5th ed.). PA, USA: Lippincott Williams \& Wilkins.

6. Daly, Walter J., and Stuart Bondurant. "Effects of oxygen breathing on the heart rate, blood pressure, and cardiac index of normal men-resting, with reactive hyperemia, and after atropine." The Journal of clinical investigation 41, no. 1 (1962): 126-132.

7. https://www.hopkinsmedicine.org/healthlibrary accessed on March 10, 2019.

8. https://www.nobelprize.org/prizes/medicine/1931/warburg/facts/. Accessed March 10, 2019.

9. https://www.aqua-angels.com/wp-content/uploads/2017/04/Warburg.pdf Accessed March 10, 2019.

10. https://www.nia.nih.gov/health/what-happens-brain-alzheimers-disease. Accessed on March 10, 2019.

11. https://lifespa.com/best-ways-detox-brain/ Accessed on March 10, 2019.

12. Delaidelli, Alberto, and Alessandro Moiraghi. "Respiration: A New Mechanism for CSF Circulation?." Journal of Neuroscience 37, no. 30 (2017): 7076-7078.

13. Olanow, C. W., and W. G. Tatton. "Etiology and pathogenesis of Parkinson's disease." Annual review of neuroscience 22, no. 1 (1999): 123-144.

14. Pissadaki, E. K., and Bolam, J. P. (2013). The energy cost of action potential propagation in dopamine neurons: clues to susceptibility in Parkinson's disease. Front. Comput. Neurosci. 7:13. doi: 10.3389/fncom.2013.00013.

15. Muddapu, Vignayanandam Ravinder Nath, Alekhya Mandali, Srinivasa Vaddadi Chakravarthy, and Srikanth Ramaswamy. "A computational model of loss of dopaminergic cells in Parkinson's disease due to glutamate-induced excitotoxicity." Frontiers in Neural Circuits 13 (2019): 11.

16. Qu, Su, Solveig Mjelstad Olafsrud, Leonardo A. Meza-Zepeda, and Fahri Saatcioglu. "Rapid gene expression changes in peripheral blood lymphocytes upon practice of a comprehensive yoga program." PloS one 8, no. 4 (2013): e61910. 